\title{
Optimising Cardiovascular Care of Patients with Multiple Myeloma
}

Marta Fontes Oliveira ${ }^{1,2}$, Willeke R. Naaktgeboren ${ }^{3,4}$, Alina Hua ${ }^{5}$, Arjun K Ghosh ${ }^{1,6}$, Heather Oakervee $^{7}$, Simon Hallam ${ }^{7,8}$, Charlotte Manisty ${ }^{1,9}$

${ }^{1}$ Department of Cardio-oncology, Barts Heart Centre, Barts Health NHS Trust, West Smithfield, London, EC1A $7 \mathrm{BE}, \mathrm{UK}$

${ }^{2}$ Cardiology Department, University Hospital Centre of Porto, Largo Prof. Abel Salazar, 4099-001 Porto, Portugal

${ }^{3}$ Netherlands Cancer Institute, Department of Psychosocial Research and Epidemiology, Plesmanlaan 121, 1066CX Amsterdam, The Netherlands

${ }^{4}$ Julius Center for Health Sciences and Primary Care, University Medical Center Utrecht, Utrecht University, Heidelberglaan 100, 3584 CX Utrecht, The Netherlands

${ }^{5}$ Department of Cardiovascular Imaging, School of Biomedical Engineering and Imaging Sciences, King's College London, St Thomas' Hospital, Westminster Bridge, 4th Floor Lambeth Wing, London, SE1 7EH UK

${ }^{6}$ Hatter Cardiovascular Institute, University College London, 67 Chenies Mews, London WC1E 6HX

${ }^{7}$ Department of Haemato-Oncology, Barts Heart Centre, Barts Health NHS Trust, West Smithfield, London, EC1A 7BE, UK

${ }^{8}$ Centre for Haemato-Oncology, Barts Cancer Institute, Queen Mary University of London

${ }^{9}$ Institute of Cardiovascular Sciences, University College London, Chenies Mews, London, UK

Key-words: multiple myeloma, cardiovascular disease, cardio-oncology

Word count: 3019 words

\section{Corresponding author:}

Marta Fontes Oliveira

Cardiology Department, University Hospital Centre of Porto

Largo Prof. Abel Salazar, 4099-001 Porto, Portugal

martafontesoliveira@gmail.com 


\section{Abstract}

Multiple Myeloma (MM) is the third most common haematological malignancy, with increasing prevalence over recent years. Advances in therapy have improved survival, changing the clinical course of MM into a chronic condition and meaning that management of co-morbidities is fundamental to improve clinical outcomes. Cardiovascular (CV) events affect up to $7.5 \%$ of individuals with MM, due to a combination of patient, disease and treatmentrelated factors, and adversely impact survival. MM typically affects older people, many with pre-existing $\mathrm{CV}$ risk factors or established $\mathrm{CV}$ disease, and the disease itself can cause renal impairment, anaemia and hyperviscosity which exacerabate these further. Up to $15 \%$ of $\mathrm{MM}$ patients develop systemic amyloidosis - with prognosis determined by the extent of cardiac involvement. Management of MM generally involves administration of multiple treatment lines over several years as disease progresses, with many drug classes associated with adverse $\mathrm{CV}$ effects including high rates of venous and arterial thrombosis alongside heart failure. Recommendations for holistic management of MM patients now include routine baseline risk stratification including ECG and echocardiography, and administration of thromboprophylaxis drugs for patients treated with immunomodulatory drugs. Close surveillance of high risk patients with collaboration between haematology and cardiology is required, with prompt investigation in the event of CV symptoms, in order to identify and treat complications early. Decisions regarding discontinuation of cardiotoxic therapies should be made in a multidisciplinary setting, taking into account the severity of the complication, prognosis, expected benefits and the availability of effective alternatives. 


\section{Introduction}

Multiple myeloma (MM) is a clonal malignant process arising from antibody-producing Blymphocytes known as plasma cells. Owing to increased diagnosis and improved survival, its prevalence is markedly increasing, making it the third most common haematological malignancy in the United States(US), where 32,270 new cases were expected in 2020.(1) Over the past decade, 'novel agents' have significantly improved remission rates and survival, evolving MM into a chronic relapsing condition. Median survival is currently nearly 6 years, and additional 'novel' therapies are poised to further improve outcomes with a third of newlydiagnosed patients now expected to live 10years or more from diagnosis. As a result, management of comorbidities and therapy-related toxicities are increasingly important to improve clinical outcomes and promote healthy survivorship in these patients.

Cardiovascular(CV) events are common in MM (up to 7.5\% reported in phase-3 trials)(2) due to a combination of patient, disease and treatment-related factors, with impact on both $\mathrm{CV}$ and MM outcomes(Figure1, Table1). Observational studies have reported more frequent cardiac events for MM patients compared to noncancer age- and gender-matched controls,(3) and increased all-cause mortality and MM-specific mortality for newly-diagnosed MM patients with co-existing CV disease.(4) Together these data underpin the importance of assessment and treatment of $\mathrm{CV}$ risk factors and disease in this population to enable safe deliver of optimal MM-directed therapy over the course of many years.

In this review, we aimed to summarize the current knowledge and provide practical considerations on the management of $\mathrm{CV}$ complications in patients with MM. 


\section{Cardiovascular risk factors}

\subsection{Patient-related factors}

MM typically affects older people, with a median age of 69years at the time of diagnosis;(5) a population already at increased baseline CV risk. It is also more than twice as common in those of black ethnicity, males (1.3:1.0) and obese patients. (5) From retrospective data, almost twothirds of newly-diagnosed MM patients had baseline CV disease, with reported prevalence of $14 \%$ for both arrhythmias and ischaemic heart disease.(6)

Rates of baseline pre-existing CV risk factors are also high (7) and may be exacerbated by the metabolic effects of treatments, particularly steroids. In a large registry of almost $8000 \mathrm{MM}$ patients, baseline prevalence of hypertension was 38\%, with significantly greater risk of developing malignant hypertension over a 2-year follow-up period compared with matched non-MM patients.(7) Data from a US multicentre registry including over $2845 \mathrm{MM}$ patients found baseline prevalence of diabetes at diagnosis to be $16 \%(8)$, rising to $>30 \%$ after glucocorticoid therapy, with evidence for worse prognosis in this group.(9)

Despite the high rates of obesity, most studies show reduced prevalence of dyslipidaemia in patients with newly-diagnosed stage-3 MM compared to controls. $(3,10)$ This has been hypothesised to be secondary to increased clearance of cholesterol by cancer cells for growth and proliferation, and it has been demonstrated in preclinical studies to reverse after treatment.(10)

\section{Disease-related factors}

\section{Renal dysfunction}

Renal dysfunction is found in almost $50 \%$ of patients at the time of diagnosis, is often of multifactorial aetiology, and frequently requires dialysis in MM patients.(11) Alongside 
increased risk for heart failure, arrhythmias and coronary events in patients with chronic impairment, acute renal failure (seen in up to $20 \%$ of MM patients, (11)) leads to fluid overload and electrolyte imbalances, increasing the potential for pulmonary oedema and arrhythmias.

\section{Anaemia}

Normocytic, normochromic anaemia is reported in $73 \%$ of newly-diagnosed MM patients, and in $97 \%$ over the course of their treatment.(12) Both bone marrow infiltration and chronic renal impairment contribute to anaemia, which in turn can lead to worsening heart failure symptoms and exacerbate pre-existing myocardial ischaemia.

\section{Hyperviscosity and thrombosis}

Hyperviscosity is commonly seen in plasma cell malignancies such as Waldenström's macroglobulinemia and in the rare IgM-secreting-MM, in which hyperviscosity has been reported in $77 \%$ cases.(13)CV complications can occur due to coronary microthrombi, with resultant myocardial ischaemia and heart failure.

MM patients are also at higher risk of developing arterial thrombosis, manifest as stroke, myocardial infarction, or peripheral artery disease. Venous thromboembolism (VTE) is common, with multifactorial mechanisms including patient comorbidities (older age, immobility exacerbated by fractures and pain), inflammation and tumour-related pro-coagulant factors. This is further exacerbated by therapy, particularly immunomodulatory drugs that are key components of current optimal front-line regimens for MM.

\section{Light chain cardiac amyloidosis}

In patients with MM (or its precursors Smouldering Myeloma and Monoclonal Gammopathy of Uncertain Significance),proliferation of an abnormal clone of plasma cells overproduces circulating light-chains which can misfold and aggregate in tissues, leading to systemic light- 
chain(AL) amyloidosis. In a cohort of newly diagnosed patients with MM, up to $15 \%$ develop overt systemic amyloidosis over the course of their disease, and nearly $30 \%$ have subclinical deposits in the subcutaneous fat, heart or liver on histology.(14) In this study, patients with incidental findings of asymptomatic amyloid did not differ in MM disease characteristics compared to those without amyloid, including whether the clone was kappa or lambda restricted. This finding emphasizes the importance of vigilance for the well-described clinical and biochemical features of amyloidosis.(14)

Of patients with systemic AL amyloidosis, $60 \%$ have cardiac involvement, with the potential for amyloid deposition within all structures of the heart, resulting in a wide spectrum of clinical manifestations.(15) Amyloid deposition within the extracellular space of the myocardium leads to restrictive pathophysiology and heart failure - initially with preserved left ventricular ejection fraction(LVEF). Circulating light-chains can also have direct toxic effects on the myocytes (proteotoxicity), leading to myocyte inflammation and apoptosis and subsequent systolic dysfunction.(16) Despite normal epicardial coronary arteries, perivascular infiltration can cause ischaemia and angina, and infiltration of the conducting system may result in conduction abnormalities requiring pacemaker implantation.(17) Atrial arrhythmias are found in half of patients,(18) with ventricular arrhythmias in $25 \% .(19)$

In all patients with known $\mathrm{MM}, \mathrm{CV}$ symptoms should prompt appropriate cardiac investigations, however diagnosis of amyloid can be challenging. ECG abnormalities (low voltages on limb leads and pseudo-infarct pattern) are highly specific (98\%) for diagnosis of cardiac amyloid, however their sensitivity is low.(20) This contrasts with echocardiography, where reduced global longitudinal strain with apical sparing pattern has high sensitivity(93\%) and specificity(82\%).(21). Red flag markers for AL amyloidosis include echocardiographic features of biventricular hypertrophy with thickening of valves, discrepancy between left ventricular(LV) wall thickness and QRS voltage, atrioventricular block, repeated elevated 
troponin levels and reduced LV global longitudinal strain with apical sparing(Figure 2). Patients with cardiac investigations suggestive of amyloidosis should undergo a myeloma screen including serum and urine electrophoresis with immunofixation and serum kappa/lambda free-light-chain quantification. Detection of a monoclonal protein suggestive of AL aetiology should prompt Haematology referral and tissue diagnosis for confirmation and typing.

Cardiac magnetic resonance incorporates tissue characterisation and has emerged as a useful diagnostic and prognostic tool in the evaluation of cardiac amyloidosis. Parametric mapping techniques using T1 measurement pre- and post-administration of gadolinium enable quantification of the extracellular volume(ECV) and provide a surrogate quantitative marker of amyloid burden. Gadolinium kinetics are abnormal, leading to difficulty nulling the myocardium, and there is generally diffuse (basal predominant) late gadolinium enhancement that may be subendocardial or transmural (Figure 2).

Outcome in systemic AL amyloidosis is primarily determined by cardiac involvement, and staging (Revised Mayo Classification) involves measurement of troponin and NT-pro-BNP, alongside serum free light-chains levels, with cardiac biomarkers also used to assess response to therapies.(22) Prognosis ranges from 6 months for stage IV patients without treatment to more than 5 years for stage I patients who undergo stem-cell-transplantation.(22)

\subsection{Treatment-related factors}

The aim of treatment in $\mathrm{MM}$ is to achieve and maintain a deep remission for the maximum duration, and patients are likely to be exposed to multiple treatment lines as their disease progresses(Figure 3). The selection and sequence of drugs used depend on an individual patient's performance status, comorbidities, response to and toxicities from previous therapies, patient preference and availability of increasingly costly 'novel' agents, which may be via 
recruitment to clinical trials. Many of these drugs have associated CV adverse effects resulting in a range of toxicities(Table 2)(23).

\section{Proteasome inhibitors}

Proteasome inhibitors(PIs) are the mainstay of MM treatment. These drugs inhibit the ubiquitin-proteasome system, leading to intracellular accumulation of aggregated proteins that is disproportionally toxic to MM cells. Given the high metabolic rate of cardiomyocytes, these cells are sensitive to the cytotoxic effects of PIs. The risk of cardiotoxicity in MM patients is about $4 \%$ with the first-in-class drug bortezomib, with higher rates in the elderly and with concomitant steroid use.(24)

Carfilzomib is a newer more potent and irreversible proteasome inhibitor which, despite reduced dose-limiting peripheral neuropathy than with bortezomib, is associated with greater risk of CV complications.(2) The phase-3 ENDEAVOUR trial reported higher rates of adverse events in the carfilzomib compared with bortezomib arms, including grade 3 hypertension and heart failure.(2) Recent prospective data from $n=95 \mathrm{MM}$ patients reported $\mathrm{CV}$ events in $51 \%$ of patients treated with carfilzomib compared with 17\% with bortezomib (any grade of severity, mainly heart failure, coronary artery events, hypertension, arrhythmia and pulmonary hypertension). Despite generally not requiring discontinuation of therapy, these events were associated with worse MM progression-free and overall survival. Of note, natriuretic peptides were highly predictive of CV complications, supporting a role for biomarker surveillance in patients receiving these treatments.(25)

Ixazomib, the first orally available proteosome inhibitor, has demonstrated a favourable cardiotoxic profile, with no differences in complications when compared to placebo,(26) suggesting that cardiotoxicity is not a drug class effect of PIs.

\section{Immunomodulatory imide drugs (IMiDs)}


IMiDs such as Thalidomide, Lenalidomide and Pomalidomide are also widely-used for patients with both newly diagnosed, relapsed and refractory MM, often in combination with PIs and corticosteroids. They act in several ways modifying the immune-microenvironment but also directly targeting the malignant cells.

IMiDs are associated with increased risk of both venous and arterial thrombosis,(27) due to their pro-coagulant and pro-inflammatory effects. Thrombosis rates rise further when IMiDs are used in combination with other MM treatments: up to 25\% with dexamethasone(28) and $58 \%$ in patients receiving dexamethasone and anthracyclines(29)].

\section{Alkylating agents}

Traditional cytotoxic chemotherapy still retains a role in the treatment of MM, with cyclophosphamide commonly used in combination with novel agents. Cardiotoxicity is rare with cyclophosphamide, but includes severe congestive heart failure(30) which usually occurs within days of administration, and is increased by other factors including high dose, combination with other cardiotoxic drugs or radiotherapy, and older age.(31) High dose melphalan is frequently given to ablate the bone marrow prior to autologous stem cell transplantation, with up to $11 \%$ of patients developing atrial fibrillation.(32) Simultaneous treatment with bendamustine, bortezomib and dexamethasone was associated with $6.5 \%$ of $\mathrm{CV}$ adverse events in a phase-2-trial including 75 patients, including heart failure and sudden cardiac death in one patient.(33)

\section{Corticosteroids}

Alongside the widely-known adverse CV effects of long-term corticosteroid use, concomitant administration with other cardiotoxic regimens may potentiate their risk. In a phase-3-trial of lenalidomide with different doses of dexamethasone, patients receiving high-dose 
dexamethasone experienced higher rates of VTE and pulmonary embolism than those receiving low-dose dexamethasone.(34)

\section{Anthracyclines}

The role of anthracyclines in treatment of MM is now predominantly reserved for aggressive relapsed extramedullary disease in younger, fitter patients. The risks of systolic dysfunction and heart failure with anthracyclines are well documented(35) and arise from interaction with topoisomerase-II $\beta$ within the cardiac myocytes leading to apoptosis, mitochondrial dysfunction and generation of reactive oxygen species. Overall incidence of anthracycline-related cardiotoxicity is widely variable, but is usually dose dependent (risk is substantially higher above cumulative dose of $240 \mathrm{mg} / \mathrm{m} 2$ doxorubicin or equivalent) with higher prevalence in patients with pre-existing CV risk factors.(35)

\section{Novel agents}

Over recent years, more effective and safer therapies are emerging for $\mathrm{MM}$ treatment. $\mathrm{CV}$ complications appear rare with these drugs in clinical trials(36-38), however most excluded patients with significant pre-existing CV disease. Daratumumab, an anti-CD38 monoclonal antibody whose use is rapidly expanding, is commonly associated with new-onset hypertension,(36) however panobinostat, a nonselective histone deacetylase inhibitor, has

minimal cardiotoxicity apart from QT prolongation (generally $<500 \mathrm{~ms}$ ) in nearly $4 \%$ of patients.(39) VTE has been observed in up to $6 \%$ of patients treated with the anti-SLAMF7 monoclonal antibody elotuzumab.(37) The recently published phase-1 DREAMM-1 trial of belantamab-mafodtin, an antibody-drug targeting B-cell maturation antigen to deliver the cytotoxic agent auristatin-F directly inside MM cells, reported no significant CV complications in heavily pre-treated patients with relapsed or refractory MM.(38) 
These novel agents do however still require long-term surveillance in real-world clinical settings, particularly if given in combination with other cardiotoxic treatments, or in patients at higher CV risk.

\section{Stem cell transplantation}

Following diagnosis and risk stratification, all patients are evaluated to determine eligibility for autologous hematopoietic stem cell transplantation(HSCT), which is proven to significantly prolong progression-free and overall survival. HSCT strategy is highly centre dependent but is not commonly offered to patients with significant comorbidities (including CV disease) due to high risk of severe complications.

HSCT survivors have a 4-fold increased risk of $\mathrm{CV}$ late effects compared to the general population.(40) This elevated CV risk depends on many factors including prior MM treatment exposure, the conditioning regimen used, and patient-related risk factors including impaired LVEF. Data on the long-term risk of LV dysfunction following HSCT are lacking. Bleeker et al. reported a clinically-significant LVEF decline in $1.6 \%$ of patients following autologousHSCT, however no association was observed with melphalan dosing and risk of cardiotoxicity.(41) A minority of patients undergo allogeneic-HSCT, which may be potentially curative, but is associated with high rates of mortality and graft-versus-host disease.

\section{Radiotherapy}

Data from patients with other cancers suggest that those receiving radiotherapy experience up to 2-fold more fatal $\mathrm{CV}$ events than non-radiated patients, and baseline $\mathrm{CV}$ risk factors, cumulative dose and age at exposure are established risk factors for radiotherapy related cardiac 
damage.(42) Up to one-third of MM patients receive radiotherapy at some point during the course of the disease. Generally, however this is locally directed at plasmacytomas and painful bony lesions, and rarely includes the heart within the high-dose field, therefore CV risk is likely lower.

\section{Practical considerations in the management of cardiac complications in patients with}

\section{Multiple Myeloma}

Given the combination of improved survival, high rates of pre-existing risk factors and CV disease, and the potential for cardiotoxicity from therapies, CV considerations should be incorporated into decision-making for MM patients.

\section{Baseline risk stratification}

All patients should undergo a baseline CV risk assessment prior to initiating or changing treatments, including a thorough history and physical examination, and optimisation of CV risk factors. An ECG and echocardiogram alongside cardiac biomarkers (troponin and NTproBNP) should be performed at baseline to identify patients with subclinical CV disease (allowing for its management and optimization) and serve as future reference if cardiac complications develop. $(43,44)$ Formal risk stratification proformas area available for patients receiving anthracyclines, PIs and IMiDs for MM treatment.(43)

\section{Surveillance and treatment of CV complications during MM therapy}

During treatment, CV symptoms should prompt immediate investigation. Evidence-based protocols for routine systematic surveillance for CV complications with particular treatment regimens are lacking. These should however be individualised - based on patient and treatmentrelated risk factors, and should include echocardiography, ECG, blood biomarkers for those at 
highest risk(Figure 4). Recently published European guidelines recommend surveillance of high-risk patients receiving anthracyclines with echocardiogram every 2-cycles of treatment and 6-months after therapy.(44)

Cancer treatment-related systolic dysfunction is considered as a decline of LVEF to below 50\% or $>10 \%$ reduction from baseline falling below the lower limit of normal, and should prompt initiation of heart failure therapy with $\beta$-blockers and angiotensin-converting enzyme inhibitors. Other parameters such global longitudinal strain (GLS) should also be considered, and a decrease falling below $-18 \%$ or a $>15 \%$ relative decrease from baseline to below the limit of normal may be considered an early sign of cardiotoxicy(44).

The decision on whether to hold or discontinue cardiotoxic therapy should be made on individual basis, taking into account the severity of the complication, the overall prognosis, expected benefits of treatment, and the availability of effective alternatives.

Statins have been associated with improved MM-related survival and overall survival in large retrospective studies,(45) but prospective studies are currently lacking. Thrombocytopenia occurs frequently during MM treatment meaning that coronary intervention in patients with coronary disease requires careful risk-benefit assessment and should balance evidence for efficacy against bleeding risks with dual-antiplatelet therapy. Similarly, for patients with atrial arrhythmias, anticoagulant choice must balance bleeding risk, platelet levels, compliance and potential drug interactions. Decision-making should be collaborative in a multidisciplinary setting involving both haematologists and cardio-oncologists, ideally with patient involvement.

\section{Thromboprophylaxis for patients treated with IMiDs}

A thromboprophylaxis strategy has been proposed by the International Myeloma Working Group and the American Society of Clinical Oncology (Figure 5),(46,47) for patients receiving IMiDs-based regimens. This should include risk stratification, with lower risk patients offered 
either aspirin or prophylactic low-molecular-weight heparin(LMWH), and LMWH or warfarin prescribed for higher-risk patients. Direct oral anticoagulants(DOACs) may be considered dependent on patient wishes, although unlicensed for this indication.(48)

\section{AL amyloidosis}

Management of AL amyloidosis involves suppressing light-chain production via treatment of the underlying plasma cell dyscrasia, and supportive treatment for CV complications. Specific evidence for benefit of standard heart failure therapies in this context is lacking, and autonomic and renal involvement commonly prevent administration.(16) Management is therefore predominantly directed towards symptomatic management of congestive heart failure with diuretics. High rates of ventricular arrhythmias and conduction abnormalities are seen early during chemotherapy, therefore pre-treatment ambulatory ECG monitoring should be considered for risk stratification, with cardiac monitoring during chemotherapy initiation for those with abnormalities. Digitalis should usually be avoided due to potential binding to the amyloid fibrils, predisposing these patients to toxicity. Implantable cardioverter defibrillators for primary prevention have been shown to be associated with high rate of appropriate shocks in a retrospective study, but no survival benefit.(49)

Newer treatments that directly target amyloid fibrils are emerging. Doxycycline has demonstrated to interfere with amyloid fibril formation and to abrogate light-chain toxicity, leading to improved survival in patients with cardiac AL amyloidosis,(50) but placebocontrolled randomised clinical trials are awaited.

\section{Conclusion}

Life expectancy is improving for patients with MM due to the emergence of new treatments and patients can live now more than a decade. High baseline CV risk in addition to MM-related disease factors and treatment cardiotoxicities lead to $\mathrm{CV}$ events in up to $7.5 \%$ of this 
population. Multidisciplinary collaboration between cardiology and haematology is recommended to minimise the risk of $\mathrm{CV}$ complications and to further improve outcomes.

\section{Patients and public involvement}

Patients or the public were not involved in the design, or conduct, or reporting, or dissemination plans of our research

\section{Funding}

MFO was awarded a EACVI training grant 2020. AH is supported by Clinical Research Training Fellowship, British Heart Foundation FS/20/13/34857. CM is supported directly and indirectly by University College London Hospitals National Institute of Health Research Biomedical Research Centre. 


\section{References}

1. American Cancer Society | Cancer Facts \& Statistics [Internet]. American Cancer Society | Cancer Facts \& Statistics. [cited 2020 Sep 27]. Available from: http://cancerstatisticscenter.cancer.org/

2. Dimopoulos MA, Goldschmidt H, Niesvizky R, Joshua D, Chng W-J, Oriol A, et al. Carfilzomib or bortezomib in relapsed or refractory multiple myeloma (ENDEAVOR): an interim overall survival analysis of an open-label, randomised, phase 3 trial. The Lancet Oncology. 2017;18(10):1327-37.

3. Kistler KD, Kalman J, Sahni G, Murphy B, Werther W, Rajangam K, et al. Incidence and Risk of Cardiac Events in Patients With Previously Treated Multiple Myeloma Versus Matched Patients Without Multiple Myeloma: An Observational, Retrospective, Cohort Study. Clin Lymphoma Myeloma Leuk. 2017;17(2):89-96.e3.

4. Mohammadi M, Cao Y, Glimelius I, Bottai M, Eloranta S, Smedby KE. The impact of comorbid disease history on all-cause and cancer-specific mortality in myeloid leukemia and myeloma - a Swedish population-based study. BMC Cancer. 2015 Nov 5;15:850.

5. Röllig C, Knop S, Bornhäuser M. Multiple myeloma. Lancet. 2015 May 30;385(9983):2197-208.

6. Kistler KD, Rajangam K, Faich G, Lanes S. Cardiac Event Rates in Patients with Newly Diagnosed and Relapsed Multiple Myeloma in US Clinical Practice. Blood. 2012 Nov 16;120(21):2916-2916.

7. Chari A, Mezzi K, Zhu S, Werther W, Felici D, Lyon AR. Incidence and risk of hypertension in patients newly treated for multiple myeloma: a retrospective cohort study. BMC Cancer. 2016 22;16(1):912.

8. La L, Jagannath S, Ailawadhi S, Durie BGM, Gasparetto CJ, Hardin JW, et al. Clinical Features and Survival Outcomes in Diabetic Patients with Newly Diagnosed Multiple Myeloma (NDMM) Enrolled in the Connect ${ }^{\circledR}$ MM Registry. Blood. 2020 Nov 5;136(Supplement 1):49-50.

9. Wu W, Merriman K, Nabaah A, Seval N, Seval D, Lin H, et al. The association of diabetes and antidiabetic medications with clinical outcomes in multiple myeloma. British Journal of Cancer. 2014 Jul;111(3):628-36.

10. Chauhan G, Schmelzer AE. A novel cholesterol/lipid delivery system for murine myeloma cell lines. Biotechnol Prog. 2017 May;33(3):795-803.

11. Yadav P, Cook M, Cockwell P. Current Trends of Renal Impairment in Multiple Myeloma. Kidney Dis (Basel). 2016 Mar;1(4):241-57.

12. Kyle RA, Gertz MA, Witzig TE, Lust JA, Lacy MQ, Dispenzieri A, et al. Review of 1027 patients with newly diagnosed multiple myeloma. Mayo Clin Proc. 2003 Jan;78(1):21-33.

13. Angelova EA, Li S, Wang W, Bueso-Ramos C, Tang G, Medeiros L, et al. IgM plasma cell myeloma in the era of novel therapy: a clinicopathological study of 17 cases. Hum Pathol. 2019;84:32134.

14. Desikan KR, Dhodapkar MV, Hough A, Waldron T, Jagannath S, Siegel D, et al. Incidence and Impact of Light Chain Associated (AL) Amyloidosis on the Prognosis of Patients with Multiple Myeloma Treated with Autologous Transplantation. Leukemia \& Lymphoma. 1997 Jan 1;27(34):315-9. 
15. Merlini G, Seldin DC, Gertz MA. Amyloidosis: Pathogenesis and New Therapeutic Options. J Clin Oncol. 2011 May 10;29(14):1924-33.

16. Falk RH, Alexander KM, Liao R, Dorbala S. AL (Light-Chain) Cardiac Amyloidosis: A Review of Diagnosis and Therapy. J Am Coll Cardiol. 2016 20;68(12):1323-41.

17. Sayed RH, Rogers D, Khan F, Wechalekar AD, Lachmann HJ, Fontana M, et al. A study of implanted cardiac rhythm recorders in advanced cardiac AL amyloidosis. European Heart Journal. 2015 May 7;36(18):1098-105.

18. Sanchis K, Cariou E, Colombat M, Ribes D, Huart A, Cintas P, et al. Atrial fibrillation and subtype of atrial fibrillation in cardiac amyloidosis: clinical and echocardiographic features, impact on mortality. Amyloid. 2019 Jul 3;26(3):128-38.

19. Sidana S, Tandon N, Brady PA, Grogan M, Gertz MA, Dispenzieri A, et al. Prognostic Significance of Holter Monitor Findings in Patients With Light Chain Amyloidosis. Mayo Clinic Proceedings. 2019 Mar 1;94(3):455-64.

20. Cheng Z, Zhu K, Tian Z, Zhao D, Cui Q, Fang Q. The findings of electrocardiography in patients with cardiac amyloidosis. Ann Noninvasive Electrocardiol. 2013 Mar;18(2):157-62.

21. Phelan D, Collier P, Thavendiranathan P, Popović ZB, Hanna M, Plana JC, et al. Relative apical sparing of longitudinal strain using two-dimensional speckle-tracking echocardiography is both sensitive and specific for the diagnosis of cardiac amyloidosis. Heart. 2012 Oct;98(19):1442-8.

22. Kumar S, Dispenzieri A, Lacy MQ, Hayman SR, Buadi FK, Colby C, et al. Revised prognostic staging system for light chain amyloidosis incorporating cardiac biomarkers and serum free light chain measurements. J Clin Oncol. 2012 Mar 20;30(9):989-95.

23. Home - electronic medicines compendium (emc) [Internet]. [cited 2020 Oct 24]. Available from: https://www.medicines.org.uk/emc\#gref

24. Xiao Y, Yin J, Wei J, Shang Z. Incidence and Risk of Cardiotoxicity Associated with Bortezomib in the Treatment of Cancer: A Systematic Review and Meta-Analysis. PLoS One [Internet]. 2014 Jan 29 [cited 2020 Sep 30];9(1). Available from:

https://www.ncbi.nlm.nih.gov/pmc/articles/PMC3906186/

25. Cornell RF, Ky B, Weiss BM, Dahm CN, Gupta DK, Du L, et al. Prospective Study of Cardiac Events During Proteasome Inhibitor Therapy for Relapsed Multiple Myeloma. JCO. 2019 Jun 12;37(22):1946-55.

26. Moreau P, Masszi T, Grzasko N, Bahlis NJ, Hansson M, Pour L, et al. Oral Ixazomib, Lenalidomide, and Dexamethasone for Multiple Myeloma. New England Journal of Medicine.

2016;374(17):1621-34.

27. Uaprasert N, Voorhees PM, Mackman N, Key NS. Venous thromboembolism in multiple myeloma: Current perspectives in pathogenesis. European Journal of Cancer. 2010;46(10):17909.

28. Weber D, Rankin K, Gavino M, Delasalle K, Alexanian R. Thalidomide alone or with dexamethasone for previously untreated multiple myeloma. J Clin Oncol. 2003 Jan 1;21(1):16-9. 
29. Baz R, Li L, Kottke-Marchant K, Srkalovic G, McGowan B, Yiannaki E, et al. The role of aspirin in the prevention of thrombotic complications of thalidomide and anthracycline-based chemotherapy for multiple myeloma. Mayo Clin Proc. 2005 Dec;80(12):1568-74.

30. Ludwig H, Miguel JS, Dimopoulos MA, Palumbo A, Garcia Sanz R, Powles R, et al. International Myeloma Working Group recommendations for global myeloma care. Leukemia. 2014 May;28(5):981-92.

31. Gottdiener JS, Appelbaum FR, Ferrans VJ, Deisseroth A, Ziegler J. Cardiotoxicity associated with high-dose cyclophosphamide therapy. Arch Intern Med. 1981 May;141(6):758-63.

32. Feliz V, Saiyad S, Ramarao SM, Khan H, Leonelli F, Guglin M. Melphalan-induced supraventricular tachycardia: incidence and risk factors. Clin Cardiol. 2011 Jun;34(6):356-9.

33. Offidani M, Corvatta L, Maracci L, Liberati AM, Ballanti S, Attolico I, et al. Efficacy and tolerability of bendamustine, bortezomib and dexamethasone in patients with relapsed-refractory multiple myeloma: a phase II study. Blood Cancer J. 2013 Nov;3(11):e162.

34. Rajkumar S, Greipp PR, Jacobus S, Callander NS, Fonseca R, Vesole D, et al. Lenalidomide plus high-dose dexamethasone versus lenalidomide plus low-dose dexamethasone as initial therapy for newly diagnosed multiple myeloma: an open-label randomised controlled trial. Lancet Oncol. 2010 Jan;11(1):29-37.

35. Henriksen PA. Anthracycline cardiotoxicity: an update on mechanisms, monitoring and prevention. Heart. 2018 Jun 1;104(12):971-7.

36. Usmani SZ, Weiss BM, Plesner T, Bahlis NJ, Belch A, Lonial S, et al. Clinical efficacy of daratumumab monotherapy in patients with heavily pretreated relapsed or refractory multiple myeloma. Blood. 2016 07;128(1):37-44.

37. Dimopoulos MA, Lonial S, White D, Moreau P, Palumbo A, San-Miguel J, et al. Elotuzumab plus lenalidomide/dexamethasone for relapsed or refractory multiple myeloma: ELOQUENT-2 followup and post-hoc analyses on progression-free survival and tumour growth. Br J Haematol. 2017 Sep;178(6):896-905.

38. Lonial S, Lee HC, Badros A, Trudel S, Nooka AK, Chari A, et al. Belantamab mafodotin for relapsed or refractory multiple myeloma (DREAMM-2): a two-arm, randomised, open-label, phase 2 study. The Lancet Oncology. 2020 Feb 1;21(2):207-21.

39. Porta-Sánchez Andreu, Gilbert Cameron, Spears Danna, Amir Eitan, Chan Joyce, Nanthakumar Kumaraswamy, et al. Incidence, Diagnosis, and Management of QT Prolongation Induced by Cancer Therapies: A Systematic Review. Journal of the American Heart Association. 6(12):e007724.

40. Chow EJ, Mueller BA, Baker KS, Cushing-Haugen KL, Flowers MED, Martin PJ, et al. Cardiovascular hospitalizations and mortality among recipients of hematopoietic stem cell transplantation. Annals of Internal Medicine. 2011;155(1):21-37.

41. Bleeker JS, Gertz MA, Pellikka PA, Larson DR, Buadi F, Dingli D, et al. Evaluation of pretransplant factors predicting cardiac dysfunction following high-dose melphalan conditioning and autologous peripheral blood stem cell transplantation. European Journal of Haematology. 2012;89(3):228-35. 
42. Bovelli D, Plataniotis G, Roila F, ESMO Guidelines Working Group. Cardiotoxicity of chemotherapeutic agents and radiotherapy-related heart disease: ESMO Clinical Practice Guidelines. Ann Oncol. 2010 May;21 Suppl 5:v277-282.

43. Lyon AR, Dent S, Stanway S, Earl H, Brezden-Masley C, Cohen-Solal A, et al. Baseline cardiovascular risk assessment in cancer patients scheduled to receive cardiotoxic cancer therapies: a position statement and new risk assessment tools from the Cardio-Oncology Study Group of the Heart Failure Association of the European Society of Cardiology in collaboration with the International Cardio-Oncology Society. European Journal of Heart Failure [Internet]. [cited 2020 Oct 13];n/a(n/a). Available from: https://onlinelibrary.wiley.com/doi/abs/10.1002/ejhf.1920

44. Čelutkienė J, Pudil R, López-Fernández T, Grapsa J, Nihoyannopoulos P, Bergler-Klein J, et al. Role of cardiovascular imaging in cancer patients receiving cardiotoxic therapies: a position statement on behalf of the Heart Failure Association (HFA), the European Association of Cardiovascular Imaging (EACVI) and the Cardio-Oncology Council of the European Society of Cardiology (ESC). Eur J Heart Fail. 2020 Jul 4;

45. Brånvall E, Ekberg S, Eloranta S, Wästerlid T, Birmann BM, Smedby KE. Statin use is associated with improved survival in multiple myeloma: A Swedish population-based study of 4315 patients. American Journal of Hematology. 2020;95(6):652-61.

46. Key NS, Khorana AA, Kuderer NM, Bohlke K, Lee AYY, Arcelus JI, et al. Venous Thromboembolism Prophylaxis and Treatment in Patients With Cancer: ASCO Clinical Practice Guideline Update. JCO. 2019 Aug 5;38(5):496-520.

47. Palumbo A, Rajkumar S V, Dimopoulos MA, Richardson PG, San Miguel J, Barlogie B, et al. Prevention of thalidomide- and lenalidomide-associated thrombosis in myeloma. Leukemia. 2008;22(2):414-23.

48. Swan D, Rocci A, Bradbury C, Thachil J. Venous thromboembolism in multiple myeloma - choice of prophylaxis, role of direct oral anticoagulants and special considerations. British Journal of Haematology. 2018;183(4):538-56.

49. Lin G, Dispenzieri A, Kyle R, Grogan M, Brady PA. Implantable cardioverter defibrillators in patients with cardiac amyloidosis. J Cardiovasc Electrophysiol. 2013 Jul;24(7):793-8.

50. Wechalekar AD, Whelan C. Encouraging impact of doxycycline on early mortality in cardiac light chain (AL) amyloidosis. Blood Cancer J. 2017 Mar;7(3):e546. 
Figure 1. Cardiovascular considerations in patients with multiple myeloma - interplay between pre-existing risk factors and disease and treatment related complications.

AL, light chain amyloidosis; LV, left ventricular
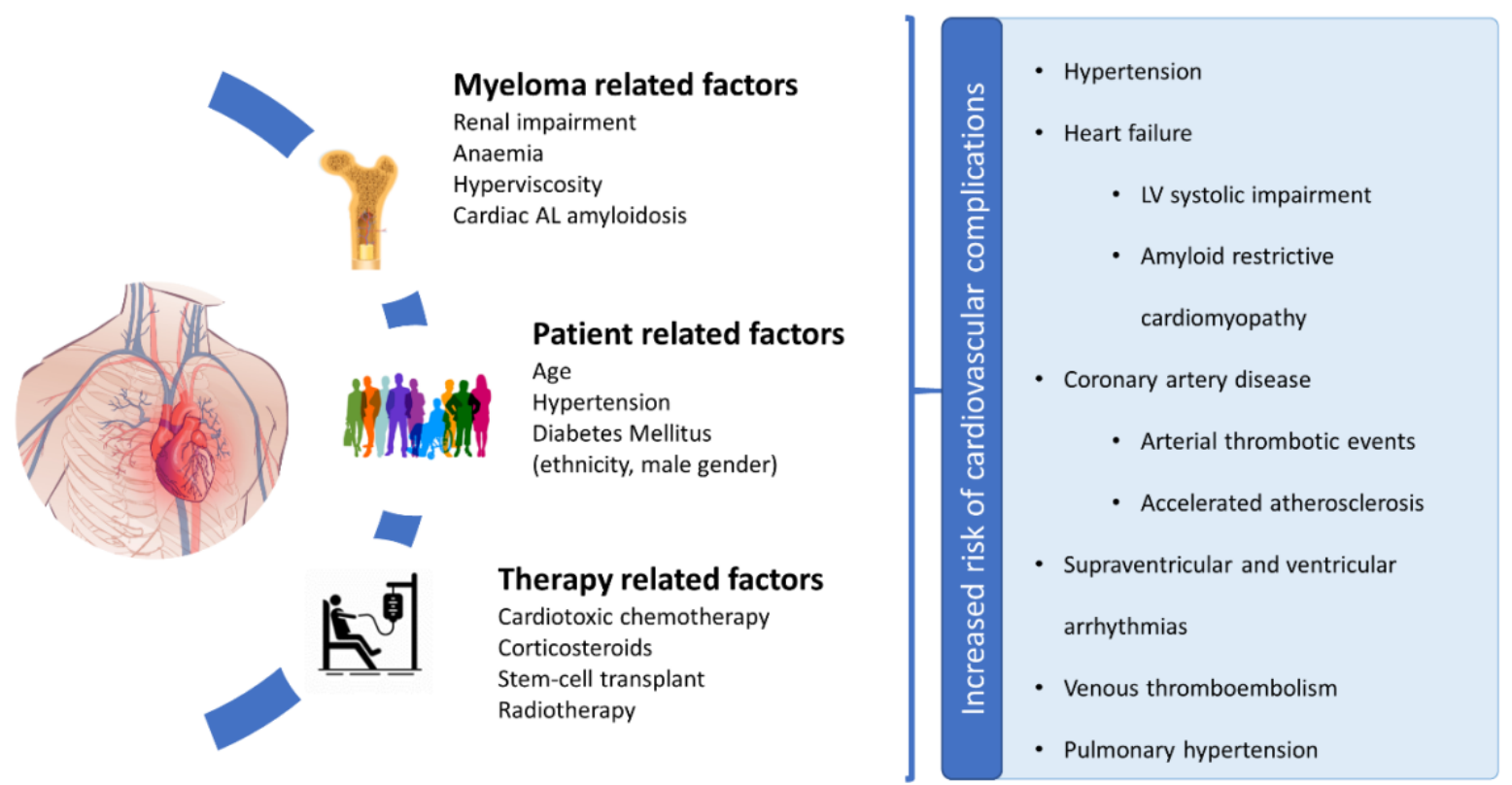
Table 1. Cardiovascular risk factors in patients with multiple myeloma

\begin{tabular}{|c|c|}
\hline Patient-related factors & Evidence \\
\hline Older age & Median age 69 years at diagnosis;(5) CV complications rise with age. \\
\hline Male predominance & MM 1.3 times more common in males $(5)$ \\
\hline Obesity & MM more common in overweight people(5) \\
\hline Black ethnicity & MM 2-3x more common with black ethnicity(5) \\
\hline Hypertension & Present in $38 \%$ at baseline, malignant hypertension in up to 4/1000 person-years in MM patients with history of hypertension (7) \\
\hline Diabetes & Present in $16 \%$ at baseline and in $>30 \%$ after glucocorticoid therapy; associated with worse prognosis $(8,9)$ \\
\hline Dyslipidaemia & Lower prevalence of dyslipidaemia in MM patients compared to controls (increased clearance of cholesterol?)(3,10,51) \\
\hline \multicolumn{2}{|l|}{ Disease-related factors } \\
\hline Renal dysfunction & $50 \%$ prevalence at diagnosis; multifactorial aetiology; associated with increased risk of acute and chronic CV complications(11) \\
\hline Anaemia & $73 \%$ prevalence at diagnosis, $97 \%$ during over the disease course (12); worsens heart failure and pre-existing myocardial ischaemia \\
\hline Hyperviscosity & Common in Waldenström's Macroglobulinemia and in IgM-secreting-MM;(13) can lead to coronary microthrombi \\
\hline Thrombosis & High risk of venous and arterial thrombosis due to comorbidities, cancer-related procoagulability, and treatments; \\
\hline Light chain amyloidosis & $\begin{array}{l}\text { Up to } 15 \% \text { of MM patients develop systemic amyloidosis; cardiac involvement leads to heart failure and arrhythmias and is } \\
\text { associated with poor prognosis(15) }\end{array}$ \\
\hline \multicolumn{2}{|l|}{$\begin{array}{l}\text { Treatment-related } \\
\text { factors }\end{array}$} \\
\hline $\begin{array}{l}\text { Proteasome inhibitors } \\
\text { (PIs) }\end{array}$ & $\begin{array}{l}\text { Associated with heart failure, coronary artery events, hypertension, arrhythmia and pulmonary hypertension;(25) carfilzomib } \\
\text { >bortezomib }\end{array}$ \\
\hline
\end{tabular}


Immunomodulatory

drugs (IMiDs)

Alkylating agents

Corticosteroids

Anthracyclines

New-novel agents

Stem cell

transplantation

Radiotherapy
Associated with increase the risk of both venous and arterial thrombosis (up to $25 \%$ in patients treated with IMiDs and dexamethasone)(28); indication for thromboprophylaxis $(27,46,47)$

Cyclophosphamide is associated with rare but severe heart failure;(30) melphalan associated with AF and supraventricular tachycardia(32)

Associated with exacerbated hypertension and diabetes, and increase CV risk; increase other drug's risk of CV complications(34) Associated with LVEF decline and heart failure(35)

Daratumumab associated with hypertension;(36) Panobinostat increases QT interval;(39) Elotuzumab associated with VTE(37) HSCT survivors have a 4-fold increased risk of CV late effects compared to the general population(40)

Increased risk of coronary artery disease and valvular disease when the heart is exposed (uncommon for MM patients)(42) 
Table 2. Cardiovascular adverse drug reactions associated with agents used to treat MM in Europe, according to the European summary of product characteristics. AMI, acute myocardial infarction; LVEF, left ventricular ejection fraction; MM, multiple myeloma; SV, supraventricular; VTE, venous thromboembolism

\begin{tabular}{|c|c|c|c|c|c|c|c|c|c|c|c|c|}
\hline \multirow{4}{*}{$\begin{array}{c}\text { Impaired LVEF / Heart } \\
\text { failure } \\
\text { Chest pain / ischaemia / } \\
\text { AMI }\end{array}$} & \multicolumn{2}{|c|}{ Proteasome inhibitors } & \multicolumn{3}{|c|}{ Immunomodulators } & \multicolumn{3}{|c|}{ Alkylating agents } & \multirow{2}{*}{$\begin{array}{c}\text { Anthracyclines } \\
\text { Doxorubicin }\end{array}$} & \multicolumn{3}{|c|}{ Newer therapies } \\
\hline & Bortezomib & Carfilzomib & Thalidomide & Lenalidomide & Pomalidomide & Cyclophosphamide & Melphalan & Bendamustine & & Daratumumab & Elotuzumab & Panobinostat \\
\hline & Uncommon & Common & Common & & & Uncommon & & Common & Common & & & \\
\hline & Uncommon & Common & Uncommon & & & Rare & & Common & & & & Uncommon \\
\hline Hypertension & Common & $\begin{array}{l}\text { Very } \\
\text { Common }\end{array}$ & & & Common & Rare & & Common & & $\begin{array}{l}\text { Very } \\
\text { Common }\end{array}$ & & Common \\
\hline Hypotension & Common & Common & & & Common & Rare & & Common & & & & $\begin{array}{l}\text { Very } \\
\text { Common }\end{array}$ \\
\hline $\begin{array}{l}\text { VTE / Pulmonary } \\
\text { embolism }\end{array}$ & Uncommon & Common & Common & Common & Common & Rare & Rare & & & & Common & \\
\hline Myocarditis & & & & & & Uncommon & & & & & & \\
\hline QT prolongation & & & & & & Rare & & & & & & Common \\
\hline $\begin{array}{l}\text { Atrial fibrillation / Atrial } \\
\text { flutter }\end{array}$ & Uncommon & Common & Uncommon & & Common & Rare & & Rare & & Common & & Common \\
\hline $\begin{array}{l}\text { SV / Ventricular } \\
\text { arrhythmias }\end{array}$ & Uncommon & & & & & Rare & & Common & Rare & & & \\
\hline Sinus tachycardia & Uncommon & Common & & & & Uncommon & & Rare & Rare & & & Common \\
\hline Bradycardia & Uncommon & & Common & & & Rare & & & Rare & & & Common \\
\hline Pericarditis & & Uncommon & & & & Rare & & & & & & \\
\hline Pericardial effusion & Uncommon & Uncommon & & & & Rare & & Uncommon & & & & \\
\hline Pulmonary Hypertension & Rare & Common & Rare & & & & & & & & & \\
\hline Cardiac arrest & Uncommon & Uncommon & & & & & & & & & & \\
\hline & & \multicolumn{11}{|c|}{ Very Common $(\geq 1 / 10)$} \\
\hline & & \multicolumn{11}{|c|}{ Common $(\geq 1 / 100$ to $<1 / 10)$} \\
\hline & & \multicolumn{11}{|c|}{ Uncommon $(\geq 1 / 1,000$ to $<1 / 100)$} \\
\hline & & \multicolumn{11}{|c|}{ Rare $(<1 / 1,000) /$ Not known } \\
\hline & & Not reported & & & & & & & & & & \\
\hline
\end{tabular}




\section{Figure 2. AL cardiac amyloidosis imaging.}

Top panel: Echocardiographic findings in patients with advanced AL cardiac amyloidosis. (A) Parasternal long axis view and showing concentric left ventricular hypertrophy (particularly noted in the interventricular septum (IVS) and posterior wall (PW)). (B) Pulse wave Doppler showing restrictive left ventricular inflow pattern (E/A ratio $>2.0$, the deceleration time is low and the e/e' ratio is elevated (the latter not shown in this picture)). (C) Strain pattern characteristic of an infiltrative process, with global reduction of the longitudinal strain sparing the apical segments. Mid and bottom panels: Cardiac magnetic resonance findings. (D) Four chamber and $(\mathrm{G})$ short axis steady state free precession cines demonstrating left ventricular hypertrophy. (H) and (E) Abnormal gadolinium kinetics with diffuse and subendocardial late gadolinium enhancement (white arrowheads). (F) Native T1 map showing a high value of T1 (reference value 970 - $1050 \mathrm{~ms}$ ). (I) Extracellular volume (ECV) map showing elevated ECV (normal <30\%). IVS: interventricular septum, LA: left atria, LV: left ventricle, PW: posterior wall, RA: right atria, $\mathrm{RV}$ : right ventricle 


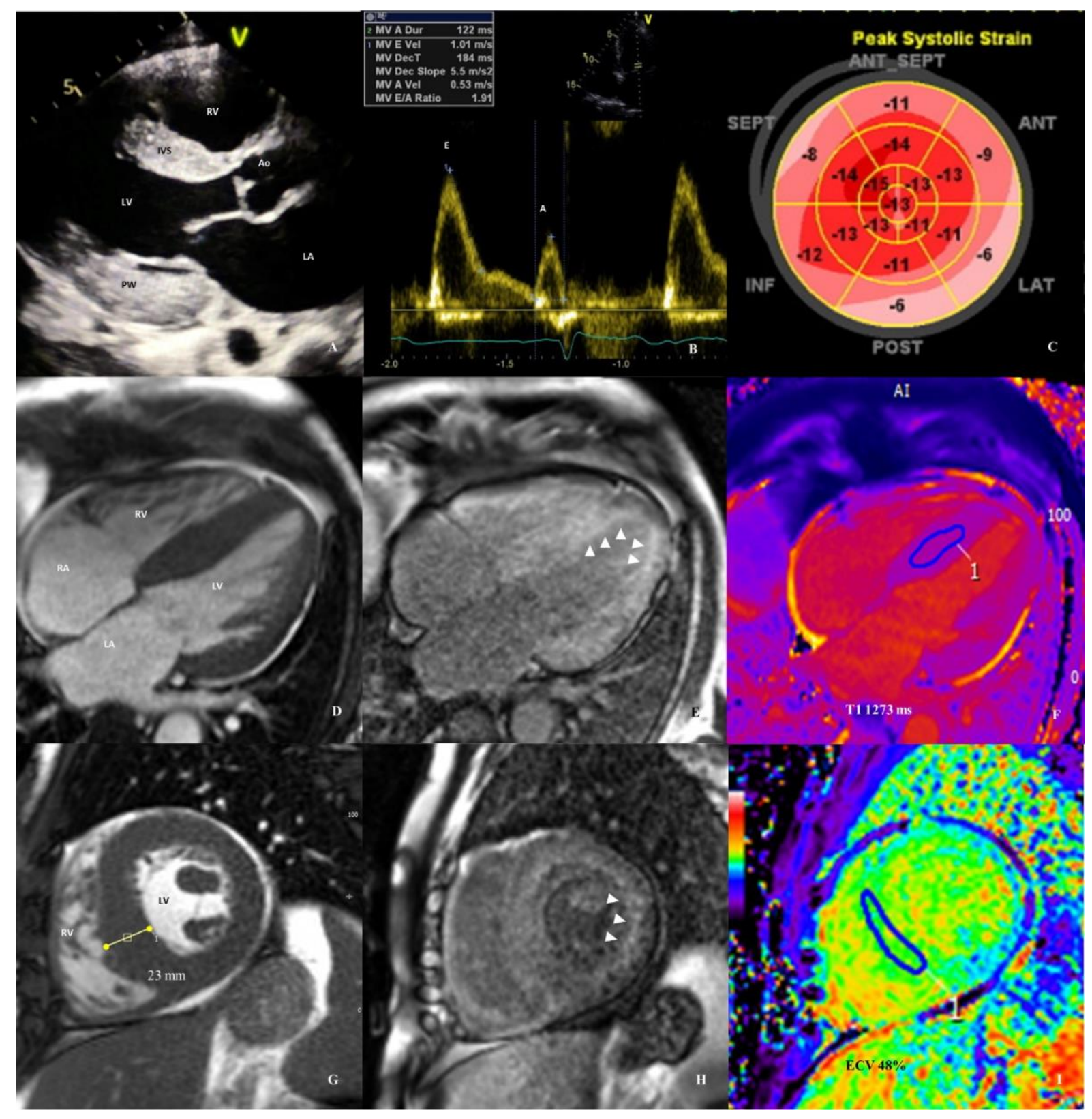


Figure 3. Agents commonly used for the treatment of Multiple Myeloma in the United Kingdom.

HSCT, hematopoietic stem cell transplantation; UK, United Kingdom

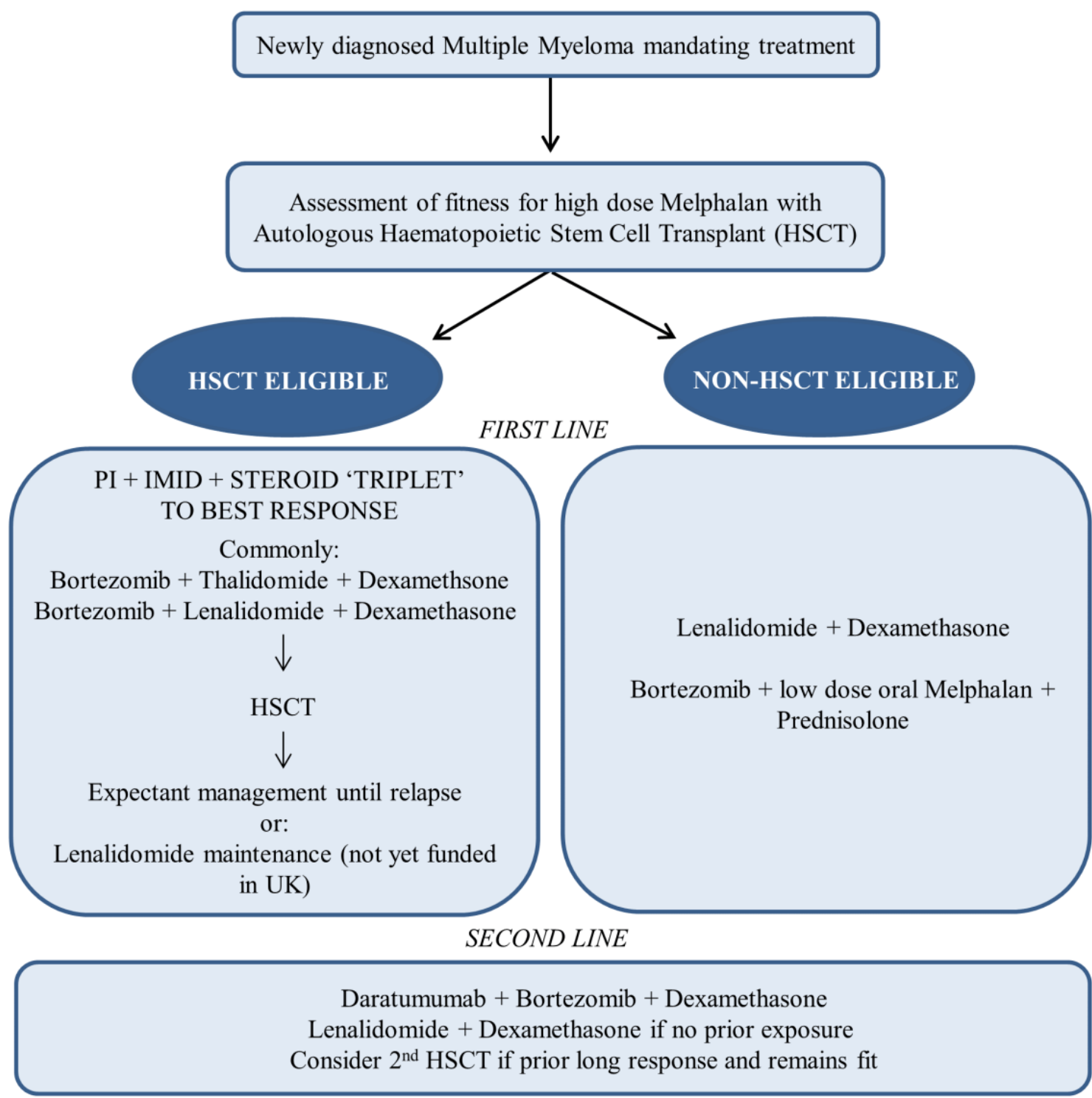

THIRD LINE

Ixazomib + Lenalidomide + Dexamethasone

Bortezomib + Panobinostat + Dexamethasone

BEYOND THIRD LINE

Strongly consider trials, drugs not previously exposed to, retreatment with previously effective drugs, or:

Pomalidomide

Daratumumab

Isatuximab + Pomalidomide +Dexamethasone 


\section{Figure 4. Cardiovascular risk assessment in MM patients and practical recommendations.}

*Recommended for all patients but essential for high risk patients. Based on the recently published position statement on baseline risk assessment in cancer patients from the European Society of Cardiology in collaboration with the International Cardio-Oncology Society (43)

** Please see Figure 4 for specific recommendations (47)

*** Please note that specific evidence for benefit of standard heart failure therapies in patients with cardiac amyloidosis are lacking.

CV, cardiovascular; ECG, electrocardiogram; IMiD, immunomodulatory drugs; LMWH, lowmolecular-weight heparin; LVEF, left ventricular ejection fraction; MM, multiple myeloma

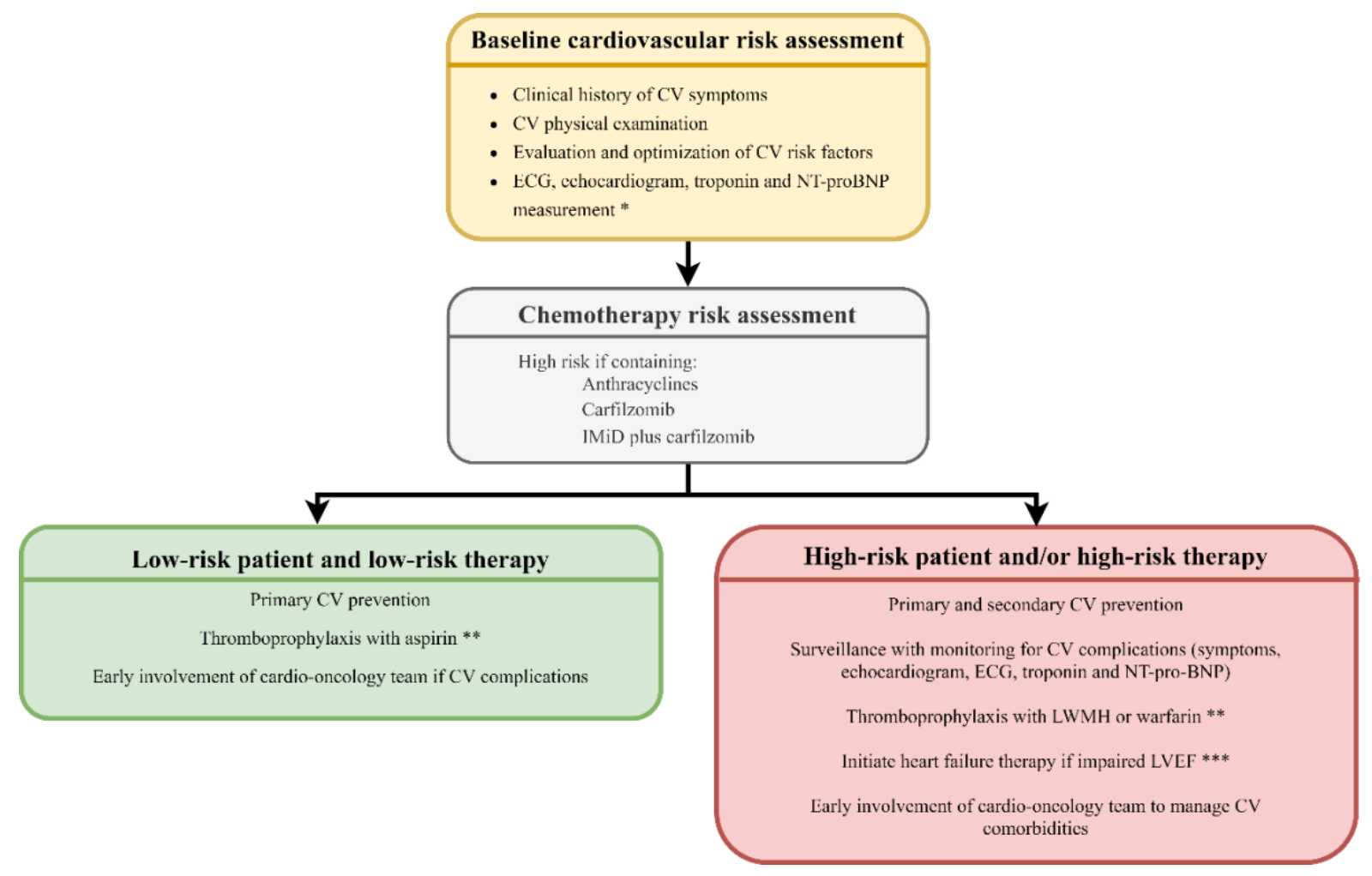




\section{Figure 5. Venous thromboembolic events (VTE) risk assessment and recommendations}

IMiD, immunomodulatory drugs; LMWH, low-molecular-weight heparin; MM, multiple myeloma; VTE, Venous thromboembolism

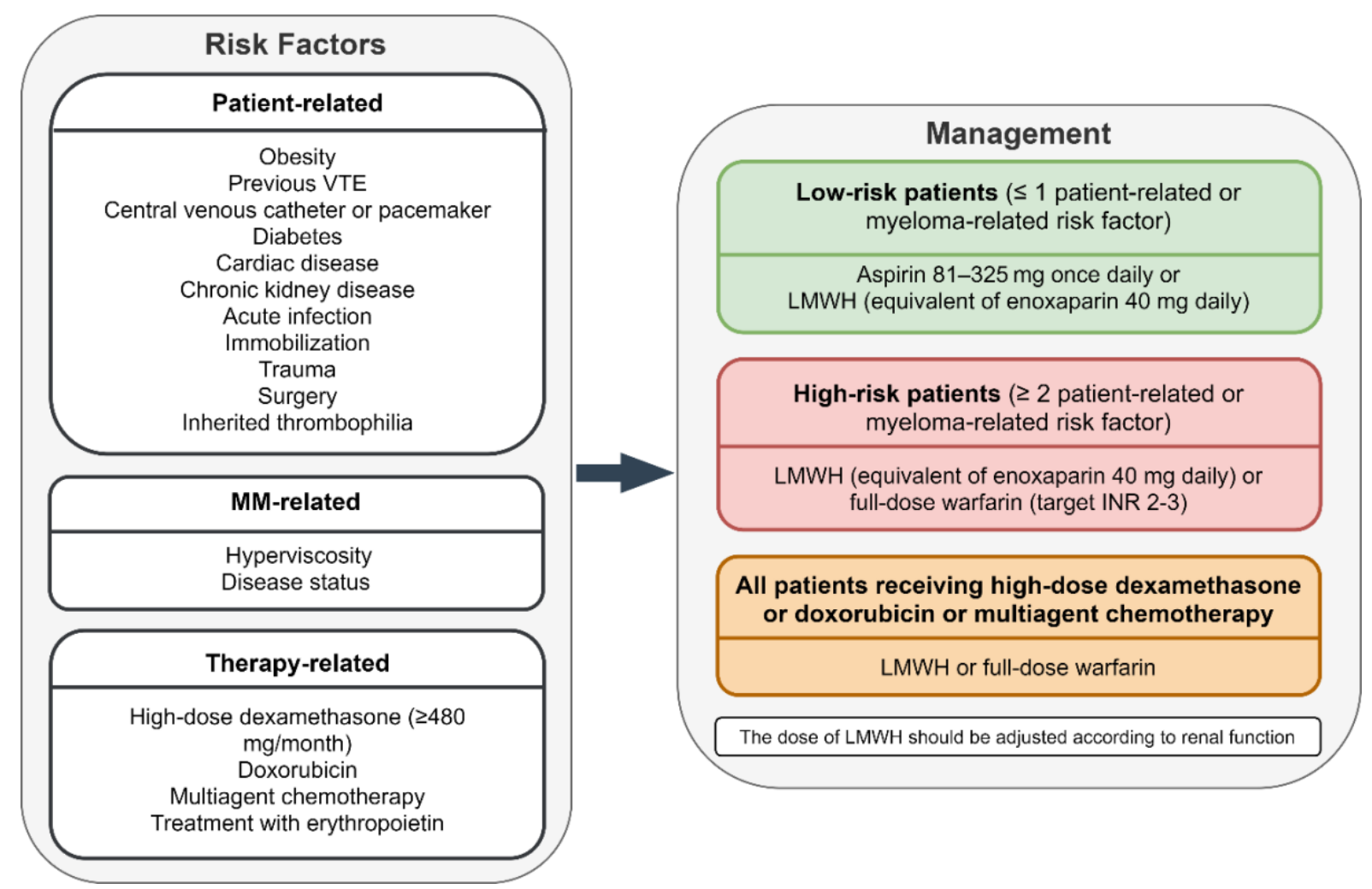

